\title{
Neonatal Septicemia: Still a Problem to Resolve
}

\author{
Amar Taksande \\ Department of Pediatrics, Jawaharlal Nehru Medical College, Sawangi (Meghe), Wardha, Maharashtra, India
}

Corresponding author: Amar Taksande, Professor, Department of Pediatrics, Jawaharlal Nehru Medical College, Sawangi (Meghe), Wardha, Maharashtra, India, Email: amar.taksande@gmail.com

Received date: May 31, 2016; Accepted date: July 18, 2016; Published date: July 25, 2016

Citation: Taksande A. Neonatal Septicemia: Still a Problem to Resolve. Prev Inf Cntrl. 2016, 2:2.

Copyright: (c) 2016 Taksande A. This is an open-access article distributed under the terms of the Creative Commons Attribution License, which permits unrestricted use, distribution, and reproduction in any medium, provided the original author and source are credited.

\section{Introdiction}

Neonatal septicemia remains significant but underestimated problem around the world and continues to be a problem in special care neonatal units [1]. There could be number of reasons for neonatal mortality and morbidity. The incidence is mainly higher in developing countries than developed countries [2]. The World Health Organization estimates that, there are about five million neonatal deaths a year, with approximately $98 \%$ occurring in developing countries [3]. Despite advances in neonatal and paediatric health care, neonatal sepsis is a significant cause of neonatal morbidity and mortality [3]. Neonatal sepsis is mainly divided into two types depending on the age of onset i.e. early onset sepsis ( $72 \mathrm{~h}$ ) and Late onset sepsis ( $\geq 72$ h-28 days). Early Onset Sepsis (EOS) is mainly acquired vertically from the genital tract of the pregnant woman before or during delivery. The incidence in EOS is about $15-19 / 1000$ live birth. The common risk factors are mainly the duration of gestation at delivery and the presence of maternal genital tract infection. In the developing countries, the most common pathogens are Escherchia coli, Klebsiella species, and Staphylococcus aureus of EOS $[4,5]$. Late Onset Sepsis (LOS) can be acquiring from the care giving environment. The incidence of LOS is about 1.87 to 5.432 per 1,000 live births. The risk factors are prematurity, central venous catheterization, nasal cannula, exposure to antibiotics and prolonged hospitalization [6]. The most common pathogens which cause LOS are Coagulase negative staphylococci, Staphylococcus aureus, Escherichia coli, Klebsiella spp. and Pseudomonas aeruginosa. It is usually acquired in the special care neonatal unit or in the community. The infections of $C$. albicans are common in preterm infants, if the neonates received prolonged antibiotics therapy [6].

The clinical diagnosis of neonatal septicemia is very difficult as it presents with non-specific signs and symptoms [7]. The clinical presentation are fever or hypothermia, poor sucking and feeding, lack of responsiveness, lethargy, irritability, hypotonia, respiratory distress, apnea, tachycardia or bradycardia, hypoglycemia and impaired peripheral perfusion. Neonates presented with respiratory distress include tachypnea, grunting, nasal flaring and intercostal retractions. These symptoms can often be the only clinical manifestation of septicemia with or without pneumonia. Pneumonia, which is more common in EOS, where meningitis and bacteremia are more common in LOS. The neonates may become complicate by the appearance of metastatic foci of infection, disseminated intravascular coagulation, shock and congestive heart failure [7]. Premature and sick neonates are more susceptible to sepsis and subtle nonspecific initial presentations. By continuous monitoring and considerable vigilance is therefore required in these neonates so that sepsis can be easily identified and treated effectively. An early diagnosis is important to initiate appropriate and prompt treatment. The correct and timely identification of infectious agents and their antibiotic sensitivity patterns are essential to guide the paediatrician regarding both the empirical and definitive treatment.

The diagnosis is usually carried out by blood, cerebral spinal fluid and urine cultures. The blood culture is the gold standard suspected case of neonatal sepsis and should always be performed before starting treatment [7]. Other diagnostic tools such as C-reactive protein, procalcitonin, mannose binding lectin, cytokine profile are being studied. The non-specific biomarkers that have been studied for diagnosis are acute phase reactant, cell-surface antigens and chemokines and cytokines [6]. The use of genomics and proteomics are rarely being analysed for detecting neonatal sepsis. It is nice to use blood culture techniques which are highly sensitive and reliable, such as the BACTEC or BacT/Alert systems [7]. Antimicrobial treatment with suspected neonatal sepsis must start immediately after birth and without delay. The reports of blood culture are not available immediately and results are not obtained in $24 \mathrm{~h}$. Because of these reasons, antimicrobial treatment is empirical started early which includes a broad converges for organisms, usually a beta lactam antibiotics and aminoglycoside. Third generation cephalosporin to be added to the empiric treatment of critically ill neonates, against the most likely pathogens [6]. After empirical treatment, the choice of the antibiotics depends on the microorganism isolated. The antibiotic therapy duration is of 10 days in EOS without meningitis, 10-14 days in LOS without meningitis and 14-21 days in cases of neonatal meningitis. The treating health worker must stop using broad spectrum antibiotics for prolonged periods otherwise resistance to antibiotics will rise. Resistance to the carbapenems, imipenem and meropenems, is already appearing high in Indian neonates, yet many bacteria are resistant to many other antibiotics. The over-use of broad spectrum antibiotics is irresponsible must be realize by the treating doctor. All 
Neonatologist and Pediatrician must make a combined and effective effort of improve prescribing practices. Thus, the knowledge of both the pathogens causing neonatal septicemia and their antimicrobial susceptibility is essential to select the appropriate anti-microbial treatment. Furthermore, the neonatal sepsis is a life threatening emergency and thus any delay in treatment may cause neonatal mortality. Prevention of healthcare associated infections through antimicrobial stewardship, early gastric feeding, limited use of invasive devices, standardization off catheter care practices, limited steroid use and proper hand washing technique are important to decrease the neonatal mortality. 


\section{References}

1. Gomaa HHA, Udo EE, Rajaram U (2001) Neonatal septicemia in AlJahra hospital, Kuwait: Etiologic agents and antibiotic sensitivity patterns. Med Princ Pract 10: 145-50.

2. Kaistha N, Mehta M, Singla N, Garg R, Chander J (2009) Neonatal septicemia isolates and resistance patterns in a tertiary care hospital of North India. J Infect Dev Ctries 4: 55-57.

3. Vergnano S, Sharland M, Kazembe P, Mwansambo C, Heath PT (2005) Neonatal sepsis: An international perspective. Arch Dis Child Fetal Neonatal 90: F220-F224.
4. Zaidi AKM, Thaver D, Ali SA, Khan TA (2009) Pathogens associated with sepsis in new-borns and young infants in developing countries. Pediatric Infectious Disease Journal 28: 10-18.

5. Rather GN, Jan M, Rafiq W, Gattoo I, Hussain SQ, et al. (2015) Morbidity and mortality pattern in late preterm infants at a tertiary care hospital in Jammu \& Kashmir, Northern India. J Clin Diagn Res 9: SC01-SC04.

6. Saez-Lopez E, Guiral E, Soto SM (2013) Neonatal sepsis by bacteria: A big problem for children. Clin Microbial 2: 125.

7. Dessì A, Pravettoni C, Ottonello G, Birocchi F, Cioglia F, et al. (2014) Neonatal sepsis. J Pediatr Neonat Individual 3: e030273. 